\title{
EL MÉTODO BIOGRÁFICO EN EL ESTUDIO DE LAS DESIGUALDADES SOCIALES
}

\author{
MARÍA JOSÉ GONZÁLEZ RÍO y BEGOÑA SAN MIGUEL DEL HOYO \\ Universidad de Alicante
}

\author{
PALABRAS CLAVE ADICIONALES ADDITIONAL KEYWORDS \\ Técnicas de investigación social, Relato de vida, Techniques of Social Research, Life Story, Life \\ Historia de vida. \\ History.
}

\begin{abstract}
RESUMEN. En este artículo se presenta una reflexión sobre el método biográfico: sus ventajas y sus limitaciones, su utilidad para el estudio de ciertas parcelas de la realidad social, las dificultades propias del análisis y la diversidad de sus modos de aplicación. Esta reflexión surge a raíz de la realización de dos investigaciones. La primera de ellas trata sobre la reconversión de los trabajadores del calzado de Elche y el paso de muchos de ellos a la economía sumergida, a través de las trayectorias laborales y vitales de 30 trabajadores. La segunda, tiene como objeto el estudio de la pobreza a través de relatos biográficos de mujeres en situación de precariedad social. Se trata de dos investigaciones distintas, pero ambas comparten el hecho de ocuparse de dos manifestaciones de los procesos de la desigualdad social.
\end{abstract}

SUMMARY. This paper makes a reflection about the biographical method in reference to: its advantages and limitations; its usefulness towards the study of certain sectors of reality; inherent analytical difficulties and the methodological diversity of application. This reflection comes off from two studies. The first one analyzed the reconversion of thirty shoe industry labourers in the city of Elche and their job path that often leads them towards the underground economy. The second study analyses poverty through the biographies of women in precarious social conditions. Both studies come together to reveal processes of social inequality.

E-mail: MJ.Gonzalez@ua.es MB.Sanmiguel@ua.es

Revista Internacional de Sociología (RIS)

Tercera Época, $\mathrm{n}^{\circ}$ 33, Septiembre-Diciembre, 2002, pp. 115-132. 


\section{INTRODUCCIÓN}

Dos investigaciones recientes, en las que hemos aplicado el método biográfico ${ }^{1}$, nos han permitido, además de revisar la bibliografia más significativa, reflexionar sobre sus ventajas y sus limitaciones, su utilidad para el estudio de ciertas parcelas de la realidad social, las dificultades propias del análisis y la diversidad de sus modos de aplicación. Este artículo no pretende, en modo alguno, constituir una monografía sobre el método biográfico - no es ésta la ocasión ni disponemos del espacio para hacer siquiera una aproximación-, sino tan sólo recoger estas reflexiones, surgidas al hilo de dos investigaciones distintas, que tienen en común el hecho de ocuparse de dos manifestaciones de los procesos de la desigualdad social.

Pretendemos eludir aquí la consideración del debate cuantitativo/cualitativo; y ello, no porque despreciemos su relevancia o porque desconozcamos sus implicaciones, sino por razones de oportunidad. Nos conformaremos con mantener que cada perspectiva se adecua en mayor medida al estudio de distintos objetos; y que, si bien es verdad que la definición teórica de dichos objetos está determinada en gran medida por los mismos presupuestos que afectan a la aplicación de los métodos, en la práctica de la investigación cabe optar por su alternancia en función de los objetos o incluso por la triangulación (Denzing, 1978), una alternativa discutible desde cierto punto de vista, pero que arroja buenos resultados en la investigación concreta.

\section{ALGUNAS CONSIDERACIONES METODOLÓGICAS}

\section{Objetos y métodos de investigación}

El método biográfico no puede circunscribirse en exclusiva al estudio de determinadas parcelas de la realidad social, como demuestra el amplio espectro de las investigaciones que se acogen a él. Sin embargo, es especialmente idóneo para la investigación de procesos de cambio social, en especial aquellos en los que se ven envueltos grupos sociales en trance de exclusión o que son empujados a situaciones de creciente marginación social. Al reconstruir la historia del método, Plummer (1989) llama la atención sobre esta cuestión: desde la inicial biografía

\footnotetext{
${ }^{1}$ Ambas investigaciones han sido ya publicadas. La primera, es de B. San Miguel, Elche: la fábrica dispersa, Instituto Juan Gil Albert, Alicante, 2001. La segunda forma parte de una investigación más amplia financiada por el Instituto de la Mujer: "Indicadores dinámicos para el estudio del empobrecimiento de las mujeres" (IM-0003) y publicada como libro por J. M. Tortosa (2002).
} 
de Thomas y Znaniecki, los sociólogos, psicólogos sociales o antropólogos que han recogido biografías se han centrado en grupos sociales pobres, marginados o en proceso de adaptación a cambios sociales muy rápidos. Así, por ejemplo, se han ocupado con frecuencia de los procesos de desviación social (Gamella, 1997); de los inmigrantes (Ramírez, 1996); de las trayectorias laborales (Bertaux, 1980; Fraser, 1970); o de sectores marginados (Negre, 1984 y Romaní, 1983).

Como señaló Becker: «A los sociólogos les gusta hablar de procesos en marcha y cosas por el estilo, pero normalmente sus métodos les impiden ver esos procesos de los que tan elocuentemente hablan» (Becker, 1966). Es de hecho la investigación basada en las historias personales, como su mismo nombre indica, la que puede obtener el debido enfoque del cambio histórico, como no es posible hacerlo con otros métodos.

El método demuestra también su idoneidad para el estudio de lo que podemos denominar "zonas de sombra" de la realidad social: aquellos aspectos de la conducta social de cualquier grupo que resultan opacos para la investigación, bien se trate de prácticas ilegales o que bordeen la ilegalidad, conductas juzgadas socialmente improcedentes o inmorales, o experiencias sociales que, por dolorosas o complejas, los sujetos difícilmente pueden comunicar. En nuestras vidas, en todas ellas, hay cuestiones sobre las que nos cuesta hablar, temores, contradicciones, problemas irresueltos, dudas. La entrevista biográfica facilita la expresión de lo latente. La idea de Bourdieu (1993) de la entrevista como "ejercicio espiritual" resulta por ello sugerente.

\section{HISTORIAS Y RELATOS DE VIDA}

Antes de adentrarnos en las consideraciones centrales sobre el método, es preciso distinguir, cuando menos, entre dos modalidades de uso de las biografias. Por una parte, la historia de vida de caso único consiste en la recogida de una sola biografía, con carácter exhaustivo y utilizando distintas fuentes. Se trata de un estudio de caso que, difícilmente, puede ir más allá de la "ejemplificación" de ciertas experiencias o ciertos sujetos. Su interés se centra en que es un caso típico - 0 atípico por extremado - de ciertas situaciones, que tiene capacidad para encarnar, $\mathrm{y}$, por tanto, puede hacer legible, un campo más vasto de experiencia. Pensemos, por ejemplo, en la obra de Lewis (1971); en esta ocasión, el procedimiento es meramente ilustrativo, ya que el caso de los Sánchez ejemplifica y concreta un campo de conocimiento: la cultura de la pobreza.

En las últimas décadas, sin embargo, muchos estudios biográficos $-\mathrm{y}$ los nuestros en particular - más que en la construcción de historias de vida, se centran en la recogida, a través de la entrevista abierta más o menos focalizada, de un número relativamente elevado de relatos biográficos — relatos de vida-, referidos a sujetos que forman parte de un campo predefinido y que, en expresión 
RIS

REVISTA INTERNACIONAL DE SOCIOLOGIA

$N^{\bullet} 33$, Septiembre-Diciembre, 2002

M² JOSÉ CONZÁLEZ RÍO y BECOÑA SAN MIGUEL DEL HOYO

de Bertaux (1993), están sometidos a condiciones socioestructurales compartidas. En este caso, la recogida de relatos biográficos tiene el carácter de una "encuesta", donde la selección de los informantes no puede ser evidentemente aleatoria, pero sí estar sujeta a cuotas que aseguren un cierto nivel de representatividad.

La posibilidad de generalización a partir del material biográfico, en el caso de los relatos de vida, se sustenta sobre la noción de "saturación", formulada por Bertaux (1993): un campo debe ser estudiado hasta el momento en que la incorporación de un nuevo caso deja de aportar novedades y resulta redundante sobre lo ya establecido anteriormente. Si es posible que se produzca "saturación de conocimiento" se debe a la existencia de "relaciones socioestructurales" particulares que organizan un medio determinado y que, por tanto, reaparecen sistemáticamente en el estudio de los casos individualizados. Puesto que son estas relaciones básicas, y no la infinita diversidad de las conductas y las percepciones individuales, lo que interesa a la investigación, la saturación se produce a partir de un número no excesivamente elevado de casos.

Es este tipo de estudios biográficos el que, a nuestro entender, tiene mayor interés para la investigación sociológica, tanto porque salva la dificultad de encontrar un sujeto dispuesto a participar en la larga y difícil tarea de construir una historia de vida, como por las posibilidades de análisis que ofrece el material producido.

\section{EL MÉTODO BIOGRÁFICO EN LA INVESTIGACIÓN SOCIOLÓGICA}

Las objeciones que se han presentado al método biográfico son ya conocidas y los defensores de los métodos cuantitativos tradicionales no se cansan de repetirlas. La primera dificultad derivaría del supuesto carácter individualizado del material que pretendemos someter a análisis. Ahora bien, no resulta difícil demostrar la sustancial ambivalencia de toda biografía, su carácter a un tiempo individual y social.

La biografía es individual porque es el sujeto quien relata una experiencia, la suya, y la interpreta. Es individual porque su trayectoria no depende exclusivamente de los escenarios sociales donde se desarrolla, sino también de decisiones que los individuos toman en función de consideraciones no sólo sociales, sino también puramente psicológicas. Es individual porque la cultura es sólo un mediador compartido en la elaboración de la experiencia. Ahora bien, la biografía es social en muchos sentidos: son sociales los escenarios donde se desenvuelve, escenarios que limitan y coartan la libertad individual, así como los procesos que determinan formas típicas de experiencia (pensemos en los cambios en el trabajo, o en las formas de provisión de servicios de bienestar social). La biografía es social porque esas experiencias son compartidas y elaboradas en grupos de referencia: familias, grupos de amigos, sindicatos, asociaciones; y también porque la 
cultura es un intermediario, pero muy eficaz, en la elaboración de la experiencia, la determinación de las expectativas y la regulación de los comportamientos.

Ahora bien, esta primera imbricación de aspectos, que, por otra parte, desconoce el resto de las técnicas de investigación sin detenerse siquiera a considerarlas, plantea a veces serios problemas al análisis sociológico. Habría que preguntarse cuáles son las condiciones bajo las que una historia individual conduce a lo social. Si una biografia es suficiente para leer una sociedad, cualquier biografía deberá serlo. Más aún, ¿todas las historias individuales, en su subjetividad absolutamente única, constituyen materia para la construcción de lo social? Ferrarotti, defensor del método, considera que: "Existe algún material biográfico más iluminador que otro; existe material biográfico que no totaliza, a un nivel visiblemente observable, ciertos aspectos de lo social; la hipótesis de leer una sociedad a través de una biografía, una específica praxis totalizadora, no es sostenible" (Ferrarotti, 1986: 122).

De este mismo carácter ambivalente del material biográfico surgen otras dificultades. El mismo Ferrarotti se ha referido a que "(...) el carácter a menudo elusivo de las declaraciones personales, expresan la ambigüedad característica de las percepciones subjetivas cortadas de sus relaciones dialécticas con un contexto estructural objetivo (...), la versatilidad titubeante de los estados anímicos, el curso imprevisible de los sentimientos" (1993: 130). También De Miguel ha llamado la atención sobre este tipo de limitaciones: "Todas las historias de vida contienen secretos, tabúes, experiencias o sucesos que nunca se cuentan, no se repiten, no se enseñan a los descendientes. Quizá exista una pauta de ocultamiento: lo que no se dice es común a muchas vidas, y, en ese sentido, es importante conocerlo sociológicamente, pues representa un modelo consistente (De Miguel, 1996: 85). En resumen, el material biográfico no está exento de la posibilidad de olvido, falseamiento o distorsión de la realidad (Hernández, 1986).

No consideramos que estas observaciones invaliden ni limiten la aplicación del método. Éstas y otras objeciones pueden ser ciertas de algún modo, pero ello no ofrece ninguna prueba de la falta de valor científico de los documentos personales (Szczepanski, 1978: 254). El mismo hecho de plantearlas, al contrario que otras metodologías, dice mucho sobre el esfuerzo reflexivo de quienes lo utilizan. El método biográfico encuentra dificultades porque pretende observar directamente la complejidad y ambivalencia de las conductas y las explicaciones elaboradas, en lugar de considerar a los actores "tontos sociales", es decir, meros soportes inertes de relaciones, u obsesos calculadores de utilidades económicas.

Cabe concluir, con Alonso (1998), que el material biográfico es susceptible de análisis sociológico: "La memoria aparece así no como una simple descripción de acontecimientos pasados, sino como la apropiación individual de una cultura histórica que siempre tiene que ser mirada desde lo colectivo". Corresponde a la investigación discernir ambos aspectos, acotar las posibilidades de generalización $\mathrm{y}$ tomar todas las cautelas necesarias. 
RIS

REVISTA INTERNACIONAL DE SOCIOLOCIA

\section{El análisis de los relatos biográficos}

Incluso si defendemos el enfoque biográfico como un instrumento válido para la investigación social, no cabe aceptar que la mera recogida de relatos biográficos constituya una tarea sociológica. De hecho, la lectura de ciertos trabajos biográficos que se dedican escuetamente a la recogida de una o varias biografías nos procuran la impresión de haber leído, simplemente, un mal relato, una mala novela. Y ello porque, ni son literatura, ni hablan del mundo social; y, si lo hacen, no es desde la sociología.

Las investigaciones que utilizan el método biográfico requieren, en primer lugar, del establecimiento de los marcos en los que se desenvuelve la trayectoria personal de los individuos. Los marcos que habitualmente son considerados en este tipo de investigaciones son los grupos primarios - la familia, los amigos, los cômpañeros- en tanto que mediadores fundamentales entre lo individual y lo social. Pero, más allá, es preciso considerar el marco histórico donde las biografias se inscriben, es decir, el conjunto de los acontecimientos y las relaciones que modelan las estructuras socioeconómicas, las instituciones donde se desenvuelve la vida de los actores y las pautas culturales. Todos esos elementos constituyen necesariamente ese marco, definen el escenario donde se desenvuelven las biografias.

El análisis sociológico de las biografias pasa, forzosamente, por la elaboración de un esquema interpretativo que organice los diversos elementos, que dé cuenta de las conductas y los discursos, que restituya el sentido social. La investigación construye así un nuevo relato en el sentido que Sennett otorga al término: "Los relatos son más que simples crónicas de los acontecimientos; dan forma al avance del tiempo, sugieren motivos que explicarían por qué ocurren las cosas, muestran sus consecuencias" (1998: 29).

Los relatos biográficos, o las historias de vida, deben ser "construidos", y esa construcción es, por mucho que el material biográfico oponga serias resistencias a nuestra intrusión, el trabajo del sociólogo. Ha de ser un trabajo que establezca las mediaciones entre uno o varios relatos biográficos y una realidad de la que pretende dar cuenta, que desmenuce la biografia y la reconstruya para dotarla de significación sociológica, tanto de los hechos que recoge, como de los modos en que los relatores piensan y elaboran dichas experiencias.

Bourdieu (1993), nada sospechoso de "subjetivismo sociológico" si atendemos a su obra, después de haber escrito contra ciertas formas de utilización del método (Bourdieu, 1989), acabó por entregar a la imprenta la trascripción de sus entrevistas desnudas de todo análisis, eso sí, precedidas de un prólogo donde, una vez más, reclama para la sociología la tarea de la interpretación y la atención a una forma de práctica de la entrevista muy alejada de sus usos más frecuentes. Desconocemos los motivos últimos de esta decisión. Es posible que el interés mismo del material recogido y su valor ilustrativo de una realidad que pretende tanto testimoniar como denunciar le indujeran a entregarlo en su estado puro, 
consciente del impacto que tendría sobre los lectores. Es posible, que considerara que la "mirada sociológica" estaba inscrita en el mismo material, en la selección y la forma de la escucha, y que el análisis escrupuloso de todas las mediaciones hubiera generado un texto demasiado prolijo, de interés sólo para profesionales. En cualquier caso, su renuncia nos ha dejado un sabor amargo a quienes compartimos muchos de sus presupuestos y sobre todo una forma de entender la investigación social.

\section{Modalidades del análisis}

Ahora bien, si reclamamos la necesidad de interpretación del material biográfico tenemos que referirnos necesariamente a los problemas que su análisis plantea. Creemos que una de las críticas más sostenibles que se han dirigido a los estudios biográficos es el carácter intuitivo de las operaciones necesarias para su análisis; y ello porque, más allá de las recetas de manual, no hay claridad sobre cómo realizar el análisis, seguramente porque no hay claridad sobre la definición de los materiales que las biografías entregan.

Los relatos biográficos, como en general la entrevista abierta, permiten acceder a un campo de conocimiento que se sitúa, en expresión de Alonso, en un terreno intermedio entre la conducta y la expresión lingüística. El material biográfico pertenece al "orden del hacer" y "al orden del decir": los sujetos hablan de "lo que hacen y lo que son (lo que creen ser y hacer)" (Alonso, 1998: 72).

En tanto que hecho de lenguaje, la entrevista biográfica no es una mera manifestación del mundo social, dado que precisamente dicha realidad está construida a través del lenguaje, que es más que una simple mediación entre la experiencia misma y su manifestación. Se trata, entonces, de un fenómeno de discurso, de una producción simbólica. Pero, a no ser que aceptemos el "paradigma lingüístico" como explicación exclusiva del mundo social, la entrevista biográfica permite explorar también las condiciones de existencia, las experiencias sociales, las prácticas, las formas de vida, es decir, el conjunto de realidades al mismo tiempo objetivas y subjetivas que constituyen la vida de los sujetos sociales, y que tienen realidad más allá del lenguaje que los expresa. En expresión acertada de Schwartz (1999) el "universo de sentido" y el "universo de vida" se anudan en los relatos biográficos y el análisis puede remitir tanto a uno como a otro; $\mathrm{o}-\mathrm{y}$ he ahí la dificultad-a ambos.

También Bertaux se ha referido a esta doble perspectiva de análisis del material biográfico: el nivel socioestructural (los modos de vida) y el nivel sociosimbólico (las actitudes, representaciones y valores individuales). En los relatos biográficos se aúnan las experiencias y descripciones del mundo social donde éstas se generan, con las elaboraciones personales y culturales de dichas experiencias. Sin embargo, estos dos niveles están inextricablemente unidos en el material biográfico; no son sino las dos caras de la misma realidad social, y por eso todo estudio ha de 
RIS

REVISTA INTERNACIONAL DE SOCIOLOCIA

№ 33, Septiembre-Diciembre, 2002

M JOSÉ GONZÁLEZ RÍO y BEGOÑA SAN MIGUEL DEL HOYO

considerar estos dos niveles simultáneamente. Ésta, creemos, es la gran ventaja de las biografías y, hay que decirlo, también su gran dificultad y el origen de esa "resistencia al análisis" que parece caracterizarlas.

Un relato biográfico puede ser leído, cuando menos, desde dos perspectivas distintas. Por una parte, estableciendo las dimensiones más materiales y concretas de la historia o de los procesos de vida de las personas entrevistadas: condiciones de existencia, experiencias, prácticas sociales, formas de vida, etc. En este caso, corresponde al análisis demostrar la pertinencia de los casos individuales, así como establecer las relaciones entre las experiencias individuales y el marco social donde se desenvuelven. La segunda perspectiva sería la del análisis del discurso - en cualquiera de sus modalidades más o menos formalizadas- que en su versión menos textual y más social atendería a la reconstrucción de la estructura de los discursos en función del contexto social. El análisis sociológico de los discursos, tal y como establece Alonso, es necesariamente histórico, remite al juego de fuerzas del campo social donde se generan "los elementos culturales y simbólicos que arman y dan consistencia a los discursos" (Alonso, 1998: 209), y, finalmente, se interesa por lo latente en la misma medida que por lo manifiesto.

Parece evidente que la perspectiva de análisis que utilicemos dependerá en gran medida de los objetivos de la investigación y, del enfoque de la investigación. Pero, igual que admitimos la posibilidad de utilizar en una misma investigación técnicas cuantitativas y cualitativas para ampliar o contrastar la información, es posible también someter el material biográfico a dos tipos de análisis. Retomando la distinción de Schwartz entre el universo de sentido y el universo de vida, y admitiendo que ambos se hallan indisolublemente ligados en el material biográfico, creemos que es la investigación la que debe definir la perspectiva a través de sus objetivos, primando una u otra, y aplicando las modalidades de análisis que sean coherentes con dichas perspectivas.

\section{LAINVESTIGACIÓN BIOGRÁFICA SOBRE TRAYECTORIAS LABORALES}

\section{Planteamiento del problema y objetivos}

Esta investigación recogió los relatos biográficos de treinta trabajadores del calzado de Elche, una localidad de la provincia de Alicante de marcada especialización industrial y que produce casi la mitad del calzado español. El primer objetivo de la investigación era conocer, a través de las trayectorias de los trabajadores o extrabajadores del calzado, cómo se había producido el proceso de reestructuración del sector que se inicia al final de la década de los setenta, y que ha transformado radicalmente el modelo industrial y de relaciones laborales en Elche y el resto de las comarcas zapateras. El segundo objetivo era conocer las consecuencias que había tenido para los trabajadores la imposición de las nuevas condiciones, 
y las formas en que se habían adaptado a las pautas actuales, especialmente en la economía sumergida, una modalidad de organización de la empresa y del trabajo que ha tenido tanta expansión en el periodo.

Este proceso había sido anteriormente estudiado, sobre todo por parte de economistas, y suficientemente descrito en relación al modelo empresarial. Sin embargo, consideramos que el estudio de las trayectorias laborales y vitales de los trabajadores podía aportar muchos elementos a su interpretación, además de revelar en detalle el modo en que los trabajadores se mueven en la economía sumergida, su adscripción a una u otra forma de trabajo, o los modos concretos de segmentación de este peculiar mercado de trabajo.

Los relatos biográficos, recogidos mediante entrevista semiestructurada, se centraban en la trayectoria laboral de los trabajadores. La estructuración parcial de la entrevista se consideró la fórmula idónea para poder conocer con detalle las biografias laborales, especialmente los sucesivos cambios de actividad o de empresa, los despidos y los mecanismos de trasvase a la economía sumergida. Interesaba, además, ampliar el conocimiento sobre la periodización del proceso y las fórmulas de inmersión de la actividad utilizadas en los distintos momentos. Por todo ello, las trayectorias laborales debían ser recogidas de forma que permitiera un cierto grado de formalización posterior.

Como ocurre inevitablemente en la situación de entrevista biográfica, el hilo de los acontecimientos narrados se ve interrumpido con explicaciones, reflexiones y referencias permanentes, tanto a la vida personal de los trabajadores como al mundo social donde se desenvuelven. Así, las entrevistas, además de las biografias individuales, reconstruyen la historia de la inmigración en Elche en la década de los sesenta, la experiencia de la inserción en el calzado de los niños inmigrantes desde los diez años, o de las mujeres que compaginaban el cuidado de los suyos con el trabajo a domicilio. Reconstruye el denso ambiente laboral de las fábricas y la dureza de las condiciones de trabajo en su interior. Reconstruye, finalmente, una parte de la historia social de la ciudad, sobre todo la de la década de los setenta, cuando el movimiento sindical y el movimiento ciudadano se habían consolidado y pudieron movilizar a miles de ciudadanos en defensa de las reivindicaciones laborales y de una demanda de derechos democráticos.

Esta perspectiva está presente, sobre todo, en las biografias masculinas. Son los hombres quienes preferentemente se ocupan del ámbito de lo público y eluden las referencias al privado, como éste y otros estudios biográficos se han encargado de probar. Las mujeres, incluso aquellas que tuvieron responsabilidades sindicales o participaron muy activamente en la organización de los conflictos laborales, se refieren, en mayor medida, a ese otro campo más próximo de las relaciones sociales: los compañeros de trabajo, la familia, los vecinos, los amigos. La fuerte especialización de ambas perspectivas según el género facilita una aproximación más compleja al estudio de un proceso que la economía o la historia han tendido a observar más limitadamente. 
RIS

REVISTA INTERNACIONAL DE SOCIOLOCIA

La densidad del material biográfico y el enorme caudal de información que aporta, tanto en éste como en otros estudios, impiden resumir en estas páginas la nómina de las informaciones y los hallazgos. Ni siquiera la investigación, necesariamente limitada en sus objetivos, es capaz de analizar la totalidad del material, viéndose obligada a despreciar una parte significativa de él. Por ello, vamos a centrarnos en el análisis de una aspecto de dicho material, aquél que por sus características consideramos que no hubiera podido conocerse por otros métodos.

\section{Relatos biográficos y encuestas laborales}

Próximo en el tiempo, se realizó otro estudio sobre el calzado. En este caso, se trataba de una encuesta dirigida en exclusiva a las mujeres trabajadoras del mismo sector en la Comunidad Valenciana. Dada la concentración de la industria del calzado en Elche, el tamaño de la muestra para esta población era muy elevado, lo que permitía obtener resultados desagregados. Puesto que los contenidos temáticos de ambos estudios eran muy similares, se contrastaron los hallazgos obtenidos a través de las distintas metodologías. Vamos a centrarnos en esta cuestión porque creemos que arroja luz sobre algunas cuestiones relevantes.

Una de las mayores discrepancias en los resultados entre la encuesta y el estudio de los relatos biográficos se refería a la trayectoria laboral de las entrevistadas: mientras que la encuesta arrojaba una visión simplificada de dichas trayectorias, con una baja incidencia de la movilidad entre los sectores formal e informal y una baja frecuencia en los cambios de empresa, las biografias retrataban unas complejísimas trayectorias laborales, cuyo análisis formalizado arrojaba resultados sorprendentes: en el plazo de una década las entrevistadas habían cambiado hasta veinte veces de situación laboral.

El análisis de esta discrepancia resulta de gran interés y revela mucho sobre la forma en que distintas técnicas producen información. En nuestro análisis, optamos por aceptar que los relatos biográficos informaban en mucha mayor medida que la encuesta sobre esta cuestión. Si tenemos en cuenta que las estadísticas oficiales establecen que la vida media de una empresa del calzado en esta última década es de 4,5 años, hay que aceptar que la trayectoria de la mayoría de las trabajadoras tiene que incluir un elevadísimo número de cambios de trabajo y de situación. ¿Cómo explicar entonces esta discrepancia? No resulta fácil resumir los argumentos, para lo que requeriríamos un espacio más amplio; sin embargo, vamos a atender a los más significativos.

En primer lugar, hay que referirse a la dificultad para memorizar, en situación de encuesta mediante cuestionario estructurado, el conjunto de la trayectoria laboral a impulso de una o dos preguntas. La complejidad de la vida de estas trabajadoras, tal y como queda reflejada en los relatos biográficos, difícilmente puede ser recogida en un cuestionario. Su vida laboral ha sido el resultado de 
profundísimas transformaciones en el sector del calzado y en el trabajo industrial en general. A partir de 1978, el trabajo ha sido inseguro por definición, especialmente en este sector, y los trabajadores han trabajado en muchas situaciones y muchas empresas distintas, tantas, que ni siquiera el relato lento y minucioso las permite evocar. La forma de recogida de información a través de cuestionario impide esta rememoración; las entrevistadas no mienten, simplemente se refieren a los pocos cambios que, espontáneamente, pueden recordar.

La segunda cuestión afecta a las categorías de construcción del cuestionario. El cuestionario no puede detenerse sobre la ambigüedad de categorías como empresa, trabajo, paro, legal, sumergido, etc., y da por hecho que los términos significan lo mismo para el investigador que para el entrevistado. Sin embargo, no es cierto. Hay que tener en cuenta que, a partir de un momento, se produjo un proceso generalizado de descentralización de la empresa hacia otras empresas, côn mucha frecuencia hacia talleres sumergidos. Estrictamente, muchos trabajadores cambiaron de empresa y de situación laboral, porque pasaron a trabajar en un taller, montado por el mismo propietario o por un intermediario, perdieron los derechos adquiridos y el estatus laboral, convirtiéndose en trabajadores sumergidos. Sin embargo, como muy bien documentan las entrevistas, los trabajadores a menudo no tienen una conciencia clara de estos cambios. Acostumbrados al cambio permanente y a la precariedad, viviendo en un medio donde estas distinciones han dejado de tener significado para quienes, simplemente, tratan de ganarse la vida, tienen dificultades para establecer el sentido de los cambios, que sólo consiguen reconstruir con la ayuda del entrevistador.

Cómo también demuestran los relatos biográficos, es muy frecuente, sobre todo en el caso de los jóvenes, que afirmen trabajar en empresas reconocidas por su marca, algunas muy prestigiosas en el mercado, cuando en realidad trabajan en talleres clandestinos que hacen una o varias fases de la producción para dichas empresas. Sin embargo, para los propios trabajadores largo tiempo socializados en la cultura de la clandestinidad, estas diferencias se difuminan hasta el punto de anular la distinción entre la empresa principal y las clandestinas subcontratadas, a pesar de las obvias consecuencias que tiene sobre las condiciones de empleo a las que están sujetos.

La tercera cuestión afectaría a las dificultades para conocer, a través del cuestionario, las prácticas ilegales o de dudosa moralidad, puesto que muchos de estos cambios de trabajo o de situación laboral tienen que ver, por ejemplo, con la práctica simultánea del cobro del seguro de desempleo y con el trabajo en el sector informal. No se trata de una voluntad expresa de mentir. Como demuestran las entrevistas biográficas, los trabajadores pueden hablar de estas prácticas, explicarlas, condenarlas o justificarlas, pero depende de en qué condiciones y delante de quién. No podemos esperar que se refieran a estas cuestiones claramente ante un encuestador anónimo, que se limita a administrar preguntas y respuestas. Pueden contarlo, y lo cuentan, en un contexto que les permita, al mismo tiempo, explicar 
RIS

REVISTA INTERNACIONAL DE SOCIOLOCÍA

№ 33, Septiembre-Diciembre, 2002

r.M.

cómo y por qué lo hicieron, ofrecer las justificaciones, reales o imaginadas, sobre su conducta. Nada muy distinto de lo que todos nosotros precisamos para poder hablar de casi todos los aspectos de nuestra vida.

Si para los trabajadores la diferencia entre clandestino o legal ha llegado a tener tan poco significado que ni siquiera pueden recordar cómo trabajaban en periodos relativamente recientes, si sólo pueden recordar que tuvieron contrato porque saben que llegaron a cobrar el seguro de desempleo, si tienen tantas dificultades para establecer su trayectoria laboral, es porque han borrado de su memoria nociones como contrato, trabajo sumergido o derechos laborales. Las encuestas no pueden dar cuenta de una experiencia que es la experiencia central de todo el proceso: la aceptación e interiorización de la precariedad laboral. De hecho, con esas categorías, y estableciendo esas diferenciaciones, sólo hablan ya dos colectivos de trabajadores: los que son o han sido sindicalistas, y los trabajadores mayores de cincuenta años enculturados en las viejas pautas del trabajo industrial.

Dilucidar completamente esta contradicción entre los distintos resultados requeriría de otras informaciones. Sin embargo, basta con retener estas tres cuestiones para demostrar que la discrepancia se halla, precisamente, en el centro de la explicación de cómo funciona el sector, en la lógica del sector mismo y, por tanto, en la lógica que ha guiado las trayectorias laborales. Los entrevistados no pueden, sencillamente, informar de dicha lógica en una entrevista por cuestionario, porque se mueven en el trabajo clandestino, porque su realidad laboral ha interiorizado hasta tal punto la clandestinidad, que sólo pueden describirla y explicarla en el discurso abierto, utilizando los recursos de la memoria, la reflexión y la elaboración de la experiencia. La encuesta enmascara, así, el problema central del funcionamiento del calzado, que no es otro que el nomadismo de los trabajadores.

\section{LA INVESTIGACIÓN BIOGRÁFICA SOBRE LA POBREZA DE GÉNERO}

\section{Objetivos de la investigación}

La recogida de relatos de vida formaba parte de un proyecto de investigación más amplio que tenía por objeto desarrollar diferentes indicadores para la medición de la desigualdad, la pobreza y la exclusión desde una perspectiva de género. Para ello, se realizó una encuesta de ámbito nacional que permitiera probar la calidad de los indicadores, así como hacer una descripción de la feminización de la pobreza en España. Sin embargo, una de las principales aportaciones del proyecto fue el estudio de la pobreza y la exclusión desde la observación del ciclo de la vida.

Son dos las razones que justifican el empleo del método biográfico y la técnica de los relatos de vida en esta investigación. En primer lugar, el objetivo de la investigación: conocer cómo viven el día a día las mujeres que se encuentran 
en situación de precariedad social. En este sentido, es habitual oír hablar en los medios de comunicación a los profesionales y a los políticos sobre los pobres, sobre la pobreza; pero sus voces, las voces de los protagonistas, rara vez se escuchan en un discurso público. Queríamos recoger los momentos clave en la trayectoria vital de las mujeres, sobre todo aquellos que han supuesto un punto de inflexión en sus vidas, conocer su vida cotidiana, sus relaciones, su formación, su trabajo, sus hijos, la vivienda y los apoyos con los que pueden contar, del mismo modo que nos interesan sus motivaciones, sus sentimientos, las razones que justifican sus decisiones. Así, podemos mirar la pobreza por dentro, conocer cómo viven las mujeres con los recursos mínimos desde su propia perspectiva.

En segundo lugar, partíamos de la consideración de la pobreza no como un estado, sino como un proceso que debe entenderse en un doble sentido: por un lado, se refiere al sentido biográfico, un proceso que se va desarrollando a largo de toda una vida y que va cambiando desde la infancia a la adolescencia, desde la edad adulta hasta la madurez; pero también, por un lado, un proceso en sentido social, económico y político. A lo largo del tiempo varían las políticas de distribución de las rentas, sube y baja el nivel de empleo, cambian las circunstancias que facilitan o restringen el acceso a la educación, a la sanidad, etc.

Así pues, consideramos que tanto la amplitud de nuestros intereses, como su naturaleza aconsejaban el empleo de esta metodología, al tiempo que hacían inviable la aplicación de una técnica estandarizada. De acuerdo con estos objetivos, la selección de las informantes se hizo siguiendo criterios teóricos y metodológicos, que nos llevaron a la configuración de una tipología en función de las variables que definen socialmente a los individuos: edad, lugar de residencia y hábitat.

El objetivo, a la hora de seleccionar a las informantes, era obtener una representación lo más amplia posible de los diferentes problemas sociales en los que se encontraban inmersas las mujeres entrevistadas. Para ello, una vez elaborada la tipología, utilizamos un muestreo de máxima variación y un muestreo de expertos, en este caso, la red de Cáritas. Finalmente, se recogieron cincuenta y siete relatos a través de entrevistas semiestructuradas a mujeres con edades comprendidas entre los 18 y 65 años.

Todas las mujeres seleccionadas en la muestra se encuentran en situación de precariedad social, aunque en circunstancias muy diferentes. Nos encontramos casos de pobreza tradicional y casos de nueva pobreza, mujeres jóvenes con cargas familiares y mujeres mayores sin cargas, mujeres que viven solas y mujeres que viven en familia, mujeres con estudios y sin estudios, mujeres con trabajo y mujeres sin trabajo, mujeres gitanas y mujeres payas; en definitiva, una representación amplia de situaciones de empobrecimiento.

\section{Los relatos biográficos}

En esta investigación las entrevistas biográficas fueron transcritas respetando las expresiones, los giros y la jerga de la informante con la mayor exactitud posi- 
ble, porque nuestro interés al realizar el análisis no se centraba únicamente en los contenidos o temas del discurso; también nos interesaba la forma misma de expresarlos, así como el sentido subjetivo atribuido. En este sentido, la información conseguida a través de los relatos es valiosa, no sólo porque nos permiten encontrar hechos y relaciones entre fenómenos, sino porque los discursos elaborados por cada una de las mujeres son, por sí mismos, explicativos de la realidad que pretendemos estudiar.

Una vez transcritas, la primera fase del análisis consistió en un trabajo de sistemátización que requiere leer los cincuenta y siete relatos con la intención de poder aislar los temas y las claves interpretativas que van apareciendo. Es una tarea comprensiva que abarca todo el material. La idea que preside esta primera etapa es la de captar toda la riqueza que encierran los discursos. Concluida esta tarea, cada investigador eligió el enfoque desde el que quería leer las entrevistas.

Descubrir los momentos y las circunstancias que hacen cambiar de forma sustancial la vida de las mujeres requiere recorrer el itinerario completo de la vida de las mujeres, reconstruir el ciclo vital de acuerdo con un línea argumental, siguiendo el eje biográfico presente, pasado y futuro. De este modo, se va esclareciendo el proceso de empobrecimiento y se puede plantear una teoría explicativa sobre el fenómeno de la pobreza. Es así como pudimos descubrir algunos datos discrepantes de los aportados por otras investigaciones, mucho más habituales, que emplean una metodología cuantitativa y una técnica de investigación como la encuesta.

Los relatos recogidos nos muestran que, si bien existen pautas y dinámicas comunes, no estamos, sin embargo, ante un colectivo homogéneo. Ciertas situaciones o acontecimientos - una familia en origen desestructurada y carente de medios económicos, la necesidad de salir del hogar familiar a una edad muy temprana, la falta de afecto o la salida del sistema educativo con un nivel de cualificación muy bajo- dan lugar a trayectorias distintas. La construcción subjetiva del pasado, la relación de unos hechos con otros, es muy diferente en cada caso. Las trayectorias se diversifican en razón de muchas variables, aunque no todas tienen el mismo peso.

En los relatos conseguimos información sobre la vida cotidiana, la vida familiar, laboral, los ingresos, los gastos, la salud o la necesidad de cuidados, las relaciones personales, familiares y sociales, las situaciones vividas y los sentimientos. Pero también surgieron otros temas, como la violencia doméstica, los problemas graves de salud, los problemas emocionales, los problemas de drogadicción y prostitución. Creemos que solamente con la realización de este tipo de entrevista biográfica es posible sacar a la luz un discurso que de otro modo quedaría silenciado.

Sin perder de vista el carácter particular de cada historia, nos encontramos también, como no podía ser de otra manera, con continuas referencias al contexto social en el que trascurren. Se ha superado la tradicional división del trabajo según la cual las mujeres se ocupaban únicamente de la realización de las tareas domésticas, mientras los hombres se ocupaban del trabajo remunerado. La sociedad 
actual exige hoy que las mujeres trabajen y adquieran independencia económica que les permita mantenerse a sí mismas y a sus familias, separarse o no casarse. Sin embargo, tanto el trabajo doméstico como el extradoméstico cuenta con un escaso reconocimiento. Los relatos ponen de relieve la situación de inferioridad de las mujeres en la sociedad y las enormes dificultades que encuentran para acceder a la educación, la sanidad, los servicios sociales y, en general, a todos aquellos medios que hacen posible el bienestar.

\section{Los resultados}

En primer lugar, los resultados de la investigación nos muestran que hay una cierta recurrencia en los factores de empobrecimiento, ninguno de los cuales, aislado, explica el resultado de la pobreza individual: la educación informal recibida, la falta de educación formal, los hábitos adquiridos (la drogadicción, el abuso en el consumo de alcohol), la mala salud heredada o adquirida por carencias alimentarias o higiénicas, son todos ellos elementos que, de una forma $\mathrm{u}$ otra, aparecen en todos los relatos.

Pero, esos factores que aparecen a lo largo de la vida no se producen todos al mismo tiempo, ni de una vez para siempre. Son factores dinámicos, aparecen y desaparecen; algunos están presentes en la familia de origen y se heredan; otros, por el contrario, se adquieren en el transcurso de la vida. Qué sucede, cómo sucede, en qué momento y cómo lo viven las mujeres, son interrogantes que requieren una respuesta que no puede conocerse a través de preguntas de cuestionario. La memoria de la entrevistada, la rapidez con la que se demanda una respuesta, las dificultades de la propia entrevistada para identificar esos factores; todo ello, unido a las limitaciones de la técnica - no olvidemos que es estática-, impiden que podamos conocer hechos de vital importancia.

Los resultados nos muestran también otra cuestión relacionada con la percepción de la pobreza. Llama la atención la alta frecuencia de mujeres a las que los entrevistadores y los Servicios de Cáritas no tenían ninguna duda en calificar como pobres y que, sin embargo, no se veían a sí mismas como tales. Como ha apuntado J. M. Tortosa en las conclusiones de la investigación, una explicación relativamente sencilla de este hecho es la necesidad de autoestima que tienen todas las personas; autoestima que, en muchos casos, se logra buscando elementos comparativos, como es la frecuencia con la que se reseña la pobreza del Tercer Mundo que se ve por televisión: ésta sí, a decir de las entrevistadas, es la verdadera pobreza, y no la situación en la que ellas se encuentran.

Lo que las entrevistas biográficas muestran es, precisamente, la complejidad de los mecanismos psíquicos y culturales que se utilizan para aceptar la propia situación y eludir, al mismo tiempo, esa responsabilización culpabilizadora, todo lo que queda oculto bajo esa sencilla declaración de pertenencia a las clases medias que recogen las encuestas. Por otra parte, no hay que olvidar que la referencia al 
Tercer Mundo, aunque forma parte sin duda de los mecanismos compensatorios, al relativizar la propia situación de carencia, es real y objetiva. En el imaginario social, además, el Tercer Mundo no sólo representa la incapacidad para asegurar la misma continuidad de la vida, sino, sobre todo, la ausencia de cualquier recurso para salir adelante. Y ese es, precisamente, el núcleo de la percepción de las mujeres sobre su presente y su futuro: que es posible enfrentar la pobreza; que es posible, a través del esfuerzo, de los estudios, del trabajo y también - cómo no- de la suerte, salir adelante, ellas mismas o, por lo menos, sus hijos.

\section{CONCLUSIONES}

Al referirnos a estas investigaciones, no hemos pretendido una descalificación de la encuesta para, por contraste, defender el enfoque biográfico en la investigación sociológica como una alternativa a sus limitaciones. No decimos que las encuestas mientan, por oposición a unos relatos biográficos que desvelarían verdades subjetivas y objetivas. Decimos, más sencillamente, que las encuestas por cuestionario no pueden decir siempre la verdad, porque hay verdades que no pueden decirse a través de respuestas estereotipadas a preguntas administradas.

Los relatos biográficos permiten comprender e interpretar los resultados de la encuesta, así como la encuesta permite enmarcar y limitar las posibilidades de generalización a partir de unos pocos relatos. El método biográfico es, entonces, una forma de aproximación a la realidad muy eficaz, sobre todo en ciertos campos, que tiene una enorme capacidad para mostrar la complejidad de dicha realidad y los múltiples factores que la conforman.

La recogida y análisis de relatos de vida referidos a personas o grupos que comparten situaciones y prácticas comunes más allá de peculiaridades idiosincrásicas, arroja luz sobre ese determinado campo de la realidad, porque pone de relieve todo aquello que es compartido, lo colectivo consciente o inconsciente, tanto en lo que se refiere a las prácticas sociales, como a su interpretación.

En definitiva, podemos afirmar que los relatos biográficos son formas de aproximación a la realidad que tienen una gran capacidad para mostrar la riqueza, la complejidad de variables que conforman la realidad social. No pueden constituir la única aproximación al conocimiento de lo social, pero son ricos en sugerencias y entregan multitud de claves para la interpretación. Finalmente, podemos concluir que, dada la complejidad de las cuestiones planteadas, es perfectamente posible orillar los problemas. Tal y como plantea Atkinson: "Carecemos de fundamentos epistémológicos y teóricos perfectos. Carecemos de métodos perfectos para la recogida de datos, carecemos de modos transparentes o perfectos de representación, trabajamos con el conocimiento de nuestros recursos limitados. Pero, no por ello, tenemos que abandonar el intento de producir relatos del mundo disciplinados, coherentes, metódicos y sensibles" (citado en Ruiz Olabuénaga, 1996:85). 


\section{BIBLIOGRAFÍA}

ALONSO, L. E. (1998), La mirada cualitativa en sociologia, Madrid, Fundamentos.

BALAN, J. (1974), Las historias de vida en las ciencias sociales. Teoria y técnica, Buenos Aires, Nueva Visión.

BECKER, H. (1966), "Introduccion", to The Jack Roller, de Clifford Shaw, Chicago, University of Chicago Press, en H. S. Becker (1971), Sociological Work, Londres, Allen Lane, pp. xiii.

BERTAUX, D. (1976), “Histoires de vie ou récit de pratiques? Méthodologie de l'approche biographique en sociologie", París, informe al Éditions L'Age d'Homme, Lausana.

(1993a), "De la perspectiva de la historia de vida a la transformación de la práctica sociológica", en J. L. Marinas y C. Santamarina (coords.), La historia oral: Métodos y Experiencias, Madrid, Debate, pp.19-34.

(1993b), "La perspectiva biográfica: validez metodológica y potencialidades", en J. L. Marinas y C. Santamarina (coords.), La historia oral: métodos y experiencias, Madrid, Debate, pp.149172.

BOURDIEU, P. (1989) “La ilusión biográfica”, Historia y Fuente Oral, vol.2, pp. 27-33.

(1993), La misere du monde, París, Éditions du Seuil.

(1995), Las reglas del arte. Génesis y estructura del campo literario, Barcelona, Anagrama.

BURKE, P. (1996), Hablar y callar. Funciones sociales del lenguaje a través de la historia, Barcelona, Gedisa.

DENZING, N. (1978), The research act: A theorical introduction to sociological methods, New York, McGraw-Hill.

DE MIGUEL, J. (1996), Autobiografias, Cuadernos Metodológicos, $\mathrm{n}^{\circ}$ 17, Madrid, CIS.

FEIXA, C. (1998), De jóvenes, bandas y tribus, Barcelona, Ariel.

FERRAROTTI, F. (1991), La historia y lo cotidiano, Barcelona, Península.

(1993), "Las biografias como instrumento analítico e interpretativo", en J.L. Marina y C. Santamarina, La historia oral: métodos y experiencias, Madrid, Debate, pp.129-139.

FRASER, R. (1970), Hablan los trabajadores, Barcelona, Nova Terra.

GAMELLA, J. F. (1997), Historia de Julián. Memorias de heroina y delincuencia, Madrid, Editorial Popular.

GIL CALVO, E. (2001), Nacidos para cambiar. Cómo construimos nuestras biografias, Madrid, Taurus. 


\section{RIS}

HERNÁNDEZ, F. (1971), "El relato biográfico en Sociología", Revista Internacional de Sociologia, vol. $44, n^{\circ} 3,2^{\mathrm{a}}$ epoca, pp. 277-293.

LEWIS, O. (1971), Los hijos de Sánchez, México, Mortiz.

MARINA, J. L. y C. SANTAMARINA (1993), La historia oral: Métodos y Experiencias, Madrid, Debate.

MARTÍN FERNÁNDEZ, M. (1994), Mujeres policía, Madrid, CIS, Siglo XXI.

NEGRE, P. (1984), Relatos de vida sobre la prostitución, Barcelona, Ayuntamiento de Barcelona.

ORTÍ, A. (2001), "En el margen del centro: la formación de la perspectiva sociológica crítica de la generación de 1956", Revista Española de Sociologia, vol.1, pp.119-168.

PLUMMER, K. (1989), Los documentos personales. Introducción a los problemas y la bibliografia del método humanista, Madrid, Siglo XXI.

PUJADAS, J. J. (1992), El método biográfico: El uso de las historias de vida en ciencias sociales, Madrid, Cuadernos Metodológicos, CIS, $\mathrm{n}^{\circ} 5$.

RAMIREZ, E. (1996), Inmigrantes en España. Vidas y Experiencias, Madrid, CIS, Monografias, $\mathrm{n}^{\circ} 147$.

ROMANÍ, O. (1983), A tumba abierta, Barcelona, Anagrama.

RUIZ OLABUÉNAGA, J.I. (1996), Metodologia de la investigación cualitativa, Bilbao, Universidad de Deusto.

SARABIA, B. (1986), "Documentos personales: historias de vida", en M. García Ferrando (comp.), El análisis de la realidad social. Métodos y técnicas de investigación, Madrid, Alianza, pp.187209.

SENNETT, R. (1998), La corrosión del carácter. Las consecuencias personales del trabajo en el nuevo capitalismo, Barcelona, Anagrama.

(2001), "La calle y la oficina: dos fuentes de identidad", en A. Giddens y W. Hutton (eds.), En el límite. La vida en el capitalismo global, Barcelona, Tusquets.

SCHWARTZ, O., C. PARADEISE, D. DEMAZIÈRE y C. DUBAR (1999), “Analyser les entretiens biographiques. L'exemple des récits d'insertion", Sociologie du Travail, $n^{\circ} 41$, pp. 453-479.

SZCZEPANSKI, J. (1978), “El método biográfico”, Revista Papers, vol.10, pp. 231-259.

TORTOSA, J. M. (2002) (Coord.), Mujeres pobres, indicadores de empobrecimiento en la España de hoy, Fundación FOESSA, Madrid. 